\title{
Las sustancias químicas y el efecto neurotóxico en los trabajadores
}

\section{(The chemical substances and the neurotoxic effect on workers)}

\author{
Rosa Morales Carrera ${ }^{1}$, Adriana Barahona Ibarra ${ }^{1}$
}

\begin{abstract}
Resumen:
Decenas de miles de trabajadores están expuestos a la contaminación ocasionada por neurotóxicos en los diferentes lugares de trabajo, en pequeños negocios, industrias artesanales e inclusive en el hogar. El problema se agrava debido, por una parte a la falta de información sobre los riesgos que presentan estas sustancias y las medidas de seguridad que deben adoptarse durante su uso, y por otro lado al exceso de confianza, que hace abstracción del peligro cuando se trata de exposiciones a pequeñas dosis de tóxico por desconocer los efectos acumulativos que producen estas sustancias cada vez que ingresan al organismo. En el Ecuador existen pocos estudios que caracterizan la exposición a estas sustancias y ninguno sobre la incidencia del síndrome neurotóxico, siendo un importante campo de investigación. Los trabajadores que están expuestos a sustancias químicas neurotóxicas se han asociado con efectos adversos en la salud humana, por su peculiar agresividad y porque comprometen la seguridad del trabajador antes de quebrantar directamente su salud. Ingresan en el organismo por vía respiratoria, dérmica o digestiva, presentan gran afinidad con las grasas por lo que se acumulan y afectan a los sistemas, órganos y tejidos grasos como el sistema nervioso central, la médula ósea y el hígado. Se han detectado efectos inmediatos (agudos) y a largo plazo (crónicos), dependiendo de la intensidad y duración de la exposición. Los síntomas son: somnolencia, pérdida del apetito, cefaleas, vértigo, depresión, ansiedad, nerviosismo, irritabilidad, fatiga, problemas de memoria, lentitud mental, convulsiones, descoordinación motora, alteraciones genéticas, entre otras.
\end{abstract}

Palabras clave: neurotóxico; plaguicidas; solventes orgánicos; metales pesados; sistema nervioso central.

\begin{abstract}
:
Tens of thousands of workers are exposed to pollution by the neurotoxicity found in their different workplaces, small businesses, handcrafting industries and even at home. The problem gets worst due to the lack of information on the risks posed by these substances and the safety controls to be taken during its use, on the other hand, the overconfidence that exists about the abstraction of this danger when it comes to the exposure to small doses of toxicity by ignoring the cumulative effects of these substances every time they enter the body. In Ecuador, nowadays there are few studies that distinguish this exposure to these substances, and none on the incidence of the neurotoxic syndrome, considering it an important field to research. Workers who are exposed to chemical toxic substances are now associated to adverse human health effects, due to its aggression and because of the worker's safety before breaking health directly. They enter the body by the respiratory, dermal or digestive system, and show a great affinity with the body grease so that it accumulates and affects the different organs, tissues, the central nervous system, the bone marrow and liver. Immediate acute and chronic long-term
\end{abstract}

1 Universidad Tecnológica Equinoccial, Facultad de Ciencias de la Ingeniería, Quito - Ecuador (rmorales@ute.edu.ec) 
effects were detected due to the intensity and duration of the exposure. Some symptoms include drowsiness, loss of appetite, headache, dizziness, depression, anxiety, nervousness, fatigue, irritability, memory problems, mental sluggishness, apathy, seizures, motor skills incoordination, genetic alterations, among others.

Keywords: Neurotoxic; insecticides; organic solvents; heavy metals; central nervous system.

\section{Introducción}

La toxicología de las sustancias químicas se manifiesta de una manera importante en la actividad laboral a partir de la revolución industrial a mediados del siglo XIX, pero es durante el siglo XX que la industria química tiene gran desarrollo y que se crea la Organización Internacional del Trabajo (OIT), que reconoce el riesgo para la salud que representan un gran número de sustancias químicas asociadas a las actividades laborales. (Enciclopedia OIT, 2012). En el listado de enfermedades laborales emitido por la OIT en el 2010, las enfermedades causadas por agentes químicos aparecen en primer lugar.

Un gran número de estudios publicados sobre efectos neurotóxicos de sustancias concretas como Plomo, Mercurio, Plaguicidas y los solventes orgánicos de tipo aromático como Benceno, Tolueno y Xileno (BTX), ponen de manifiesto este hecho, tomando en cuenta que hay más de 100.000 sustancias químicas en uso industrial o comercial que se pueden considerar tóxicas. (Olmos, M., Fundación MAPFRE, 2011).

Las investigaciones más relevantes y que han servido para definir los niveles de toxicidad en cuanto a BTX, se encuentran nombradas en el documento del Instituto Nacional de Seguridad e Higiene del Trabajo INSHT, la más antigua se remonta a 1975, "A niveles mayores de exposición, del orden de 90-150 ppm (345-576 mg/m3), se han detectado en diversos ensayos basadas en los resultados de las pruebas de rendimiento y síntomas de mareos, cefaleas e irritación de la membrana mucosa" (Stewart et al., 1975; Dick et al., 1984; Baelum et al., 1985; Echeverría et al., 1989 citado en DLPE DEL INSHT, 2007).

Así también, "en un trabajo del National Institute for Occupational Safety and Health (NIOSH), de los Estados Unidos, se incluían las alteraciones nerviosas como uno de los 10 grupos principales de enfermedades de origen laboral, y se estimaba, de manera conservadora, que cerca de 8 millones de trabajadores de este país estaban expuestos durante toda su jornada laboral a alguna sustancia neurotóxica" (Vela, M., Laborda, R., \& García, A. M. ,2003).

En el Ecuador el listado de enfermedades profesionales se encuentra en el Registro Oficial, Suplemento Año III №599 del lunes 19 de diciembre de 2011, en el cual el Instituto Ecuatoriano de Seguridad Social IESS, mediante resolución C.D. 390, expide el Reglamento del Seguro General de Riesgos del Trabajo, que en el Capítulo 1 Art 12 dice: "Se considerarán enfermedades profesionales u ocupacionales las publicadas en la lista de la Organización Internacional del 
Trabajo, OIT, así como las que determinare la Comisión de Valuación de Incapacidades, CVI...”. El primer Anexo de dicho suplemento, corresponde al listado de enfermedades profesionales, en el punto 1.1 constan aquellas causadas por agentes químicos.

En la industria ecuatoriana el uso de sustancias químicas neurotóxicas, sobre todo, plaguicidas y solventes aromáticos así como de productos que los contienen es frecuente en muchos campos laborales a nivel industrial y artesanal (Borgel, 2011); sin embargo, pocos son los estudios que existen y que determinan el riesgo por exposición a estas sustancias. Y no existen estudios sobre la incidencia de síndrome neurotóxico en los trabajadores.

En el año 2007 el Ilustre Municipio del Distrito Metropolitano de Quito, preocupado por los niveles de contaminación provocados por el parque vehicular de la ciudad, impulsa el estudio denominado "DMQ Ambiente y Salud" realizado por la Corporación IFA, en el que se determinó la presencia de compuestos orgánicos volátiles en el aire y la exposición en algunos grupos poblacionales, desde el punto de vista laboral, es importante destacar el caso de los trabajadores informales o comerciantes, en quienes se encontró afectación sobre todo en sectores de alto tráfico y congestión vehicular (La Marín, La calle Necochea y Cumbayá), obteniéndose resultados de la determinación de benceno en orina elevados, por ejemplo, en horas pico en Cumbayá (2,7 ug/l) respecto al de los grupos de control (0,1 ug/l) (Harari y cols, DMQ-Corporación IFA, 2007).

En el 2011 se realiza el estudio "Condiciones de Trabajo y derechos laborales en la floricultura ecuatoriana" que en el punto 2.2.3. trata sobre la Salud de los Trabajadores, realiza una evaluación de la exposición a plaguicidas, determinándose que está presente en varios puntos del proceso productivo. (Harari y cols, Corporación IFA, 2011)

Se considera relevante la siguiente puntualización que realizan los autores del estudio: "El hecho de que no siempre se presenten intoxicaciones agudas importantes, sino leves, no debe ser motivo de subestimación de la exposición crónica a baja dosis a los plaguicidas en la floricultura" (Corporación IFA, 2011). Esto sucede sobre todo en trabajos artesanales o informales en los que generalmente los trabajadores no dan importancia a las exposiciones a bajas concentraciones y no adoptan las medidas de prevención necesarias, y los síntomas leves como cansancio, dolor de cabeza entre otros son atribuidos a otras causas (stress) llegando así a una intoxicación crónica irreversible. Un ejemplo representativo de esto es el desastre ocurrido en la Bahía de Minamata (Japón), una población de pescadores, en el que una industria petroquímica vertía al mar los residuos tóxicos que contenían mercurio y la población estuvo expuesta por alrededor de 20 años a pequeñas dosis de mercurio que eran ingeridas en el pescado, hasta que aparecieron síntomas crónicos de intoxicación evidenciándose inclusive alteraciones genéticas del sistema nervioso central en los niños que nacían de madres expuestas. (Rodríguez, G., 2011). 
Son de especial interés en esta revisión los problemas generados por los solventes orgánicos aromáticos benceno, xileno y tolueno (BTX), respecto de los cuales existen varios estudios que demuestran la incidencia del Síndrome neurotóxico por exposición a estas sustancias, entre los más relevantes tenemos:

- El estudio "Evaluación psicológica en trabajadores expuestos a tolueno en una empresa mexicana de autopartes" (Almirall,P., 1999), el análisis se realizó en 20 trabajadores expuestos a Tolueno, adscritos al Departamento de Asbesto y 10 trabajadores no expuestos como control, que pertenecían a los Departamentos de: Juntas Blandas, Almacén, Taller mecánico y Moldeado. El trabajo concluye que existen evidencias claras de alteraciones neurotóxicas en trabadores expuestos a los solventes en relación a trabajadores no expuestos.

- "Efectos crónicos neurocomportamentales en trabajadores del sector petrolero expuestos a solventes orgánicos aromáticos (benceno, tolueno y xileno- btx)" (Castellar, M., 2007) determina una fuerte correlación entre la exposición a solventes aromáticos con déficits neurocompartamentales, evidenciándose como efectos principales trastornos de la función cognitiva como pérdida o deterioro de la memoria reciente, dificultad de concentración y atención.

- En el artículo "Alteraciones neuropsicológicas en trabajadores expuestos a neurotóxicos", se concluye que existen fuertes evidencias de alteraciones neuroconductuales en trabajadores expuestos a tolueno y xileno, incluso en rangos inferiores a los permitidos, refiere por ejemplo dos estudios:

Uno, realizado en 30 mujeres expuestas a tolueno durante 5 años, en una ensambladora de equipos electrónicos, las cuales presentaron alteraciones como disminución para destreza manual y memoria de dígitos, la media de exposición ponderada en el tiempo (TWA) fue de 88 ppm. Y el otro, en 30 trabajadores de una prensa de rotograbado con una exposición a 40 ppm durante 29 años. (Maizlish, 1994). En la hoja informativa para Tolueno de Agencia para Sustancias Tóxicas y el Registro de enfermedades (ATSDR) consta que "la Administración de Salud y Seguridad Ocupacional (OSHA) ha establecido un límite de 200 partes de tolueno por millón de partes de aire en el área de trabajo (200 ppm)". (ATSDR, 2001)

El uso de Benceno, Tolueno y Xileno (BTX) es frecuente en Ecuador, ya sea como materia prima o en productos terminados, tanto a nivel industrial, artesanal e incluso doméstico. No se han determinado con exactitud las actividades relacionadas con estas sustancias, especialmente aquellas en que las dosis de exposición serían bajas, como por ejemplo: trabajos de restauración de obras de arte (Gherardi, 2007; Sacristan, 2003), zapaterías, artistas (pintores y escultores), carpinteros, etc. En las actividades mencionadas tampoco se ha caracterizado la exposición, ni se conoce la incidencia de alteraciones crónicas, considerándose un importante campo de estudio. 


\section{Neurotoxicidad}

Simonsen y otros (1994), han trabajado sobre el concepto de neurotoxicidad y los problemas para la clasificación de los neurotóxicos. Definen la neurotoxicidad como "la capacidad de inducir efectos adversos en el sistema nervioso central, sistema nervioso periférico o los órganos de los sentidos. Se considera que un producto químico es neurotóxico cuando es capaz de inducir un patrón constante de disfunción neural, así como cambios en la química o estructura del sistema nervioso".

En estudios realizados en trabajadores expuestos a metales pesados (plomo, mercurio, cadmio), plaguicidas y solventes orgánicos (benceno, tolueno, xileno) en las industrias químicas se ha determinado que estos productos provocan síndromes neurotóxicos que pueden volverse irreversibles y presentan síntomas como falta de coordinación y de memoria, intolerancia al alcohol, afecciones renales, vértigos y cefaleas (Agency for toxic sustances \& disease registry ATSDR 2001).

Simonsen y otros (1994) en su estudio Methodological approach to the evaluation of neurotoxicity data and the classification of neurotoxic chemicals, realizan un ordenamiento de efectos mencionados en la OIT de acuerdo con el tiempo de exposición y la dosis, asignando una escala 1 a 6, como se puede observar en la tabla 1. Cuando las exposiciones son de corta duración pueden causar síntomas subjetivos como los mareos y cefaleas, pero en este grupo los efectos son reversibles; si la dosis sigue en aumento se presentan alteraciones neurológicas y en ocasiones alteraciones morfológicas irreversibles y en los niveles 3, 4, 5 ó 6 ya se manifiestan alteraciones de la química o la fisiología del sistema nervioso que suelen ser constantes.

Tabla 1. Efectos neurotóxicos según la clasificación de Simonsen y cols.

\begin{tabular}{|c|l|l|}
\hline NIVEL & \multicolumn{1}{|c|}{ GRUPO } & \multicolumn{1}{|c|}{ EFECTOS NEUROTÓXICOS } \\
\hline 6 & $\begin{array}{l}\text { Alteraciones } \\
\text { morfológicas }\end{array}$ & $\begin{array}{l}\text { Muerte celular, axonopatía, y alteraciones morfológicas } \\
\text { subcelulares }\end{array}$ \\
\hline 5 & $\begin{array}{l}\text { Alteraciones } \\
\text { neurológicas }\end{array}$ & $\begin{array}{l}\text { Hallazgos anómalos en exploraciones neurológicas en } \\
\text { seres humanos. }\end{array}$ \\
\hline 3 & $\begin{array}{l}\text { Alteraciones fisiológicas } \\
\text { o del comportamiento }\end{array}$ & $\begin{array}{l}\text { Hallazgos experimentales en animales o seres humanos } \\
\text { por ejemplo variaciones en los potenciales evocados o } \\
\text { electroencefalograma o alteraciones en pruebas } \\
\text { psicológicas o del comportamiento). }\end{array}$ \\
\hline 2 & $\begin{array}{l}\text { Alteraciones } \\
\text { bioquímicas }\end{array}$ & $\begin{array}{l}\text { Alteraciones de parámetros bioquímicos importantes (por } \\
\text { ejemplo en el nivel del transmisor, el contenido de proteína- } \\
\text { AFG o en actividades enzimáticas). }\end{array}$ \\
\hline 1 & $\begin{array}{l}\text { Síntomas subjetivos } \\
\text { irreversibles }\end{array}$ & $\begin{array}{l}\text { Síntomas subjetivos. Ausencia de indicios de anomalías en } \\
\text { las exploraciones neurológica, psicológica o cualquier otra } \\
\text { exploración médica. }\end{array}$ \\
\hline Síntomas subjetivos & $\begin{array}{l}\text { Síntomas subjetivos. Ausencia de indicios de anomalías en } \\
\text { las exploraciones neurológica, psicológica o cualquier otra } \\
\text { exploración médica. }\end{array}$ \\
\hline
\end{tabular}


El efecto neurotóxico puede ser agudo o crónico, los síntomas varían de acuerdo con el grado de exposición, el tipo de sustancia y la sensibilidad de la persona.

\section{Intoxicación Aguda}

Los síntomas se agravan dependiendo de la cantidad de sustancia neurotóxica a la que se haya expuesto, los efectos agudos son inmediatos. Si la exposición ha sido leve los efectos serán transitorios y pueden desaparecer al cesar la exposición. Se manifiestan con cefaleas, mareos, cansancio, irritabilidad, vértigo, euforia, irritabilidad, traumatismos, esguinces, quemaduras, lesiones oculares. Si la exposición es a una alta concentración puede dar lugar inclusive a la muerte. La intoxicación aguda, se trata de un suceso súbito y violento y por lo tanto, se considera como accidente de trabajo. (Cortés, 2007; Nolla, 2001)

\section{Intoxicación Crónica}

La exposición constante a niveles bajos o medios de sustancias neurotóxicas durante algún tiempo puede alterar la fisiología del sistema nervioso progresivamente, presentando patologías por inhalación o por contacto que suelen ser más graves con presencia de alteraciones de órganos y sistemas específicos, asma, producción de cáncer, modificaciones hereditarias o malformaciones en la descendencia. La intoxicación repetitiva de una misma sustancia deriva en enfermedad profesional. (Cortés 2007; Nolla 2001)

\section{Clasificación de las sustancias químicas neurotóxicas}

Es fundamental conocer cuáles son las sustancias neurotóxicas y los efectos neurotóxicos que se manifiesta dependiendo de factores como el tiempo de exposición, la naturaleza de la sustancia, la dosis y la sensibilidad de la persona expuesta (tablas 2, 3 y 4).

Tabla 2. Gases asociados a efectos neurotóxicos

\begin{tabular}{|l|l|l|l|}
\hline $\begin{array}{l}\text { Producto } \\
\text { químico }\end{array}$ & $\begin{array}{l}\text { Ejemplos de fuentes } \\
\text { de exposición }\end{array}$ & $\begin{array}{l}\text { Selección de } \\
\text { industrias con } \\
\text { riesgo }\end{array}$ & Efectos \\
\hline $\begin{array}{l}\text { Anhídrido } \\
\text { carbónico } \\
\left(\mathrm{CO}_{2}\right)\end{array}$ & $\begin{array}{l}\text { Soladura; fermentación; } \\
\text { fabricación, } \\
\text { almacenamiento y uso } \\
\text { de hielo seco }\end{array}$ & $\begin{array}{l}\text { Industria } \\
\text { metalúrgica; } \\
\text { minería; fábricas } \\
\text { de cervezas. }\end{array}$ & $\begin{array}{l}\text { M: Vasodilatación } \\
\text { A: Cefaleas; disnea; temblor, pérdida } \\
\text { de conciencia } \\
\text { C: Prácticamente ninguno }\end{array}$ \\
\hline $\begin{array}{l}\text { Monóxido } \\
\text { de } \\
\text { carbono } \\
(\mathrm{CO})\end{array}$ & $\begin{array}{l}\text { Reparación de coches; } \\
\text { soldadura; fundición de } \\
\text { metales; conductores; } \\
\text { bomberos }\end{array}$ & $\begin{array}{l}\text { Industria } \\
\text { metalúrgica; } \\
\text { minería; } \\
\text { transportes; } \\
\text { centrales } \\
\text { eléctricas. }\end{array}$ & $\begin{array}{l}\text { M: Privación de oxígeno } \\
\text { A: Cefaleas; somnolencia; pérdida } \\
\text { de conciencia }\end{array}$ \\
\hline
\end{tabular}




\begin{tabular}{|c|c|c|c|}
\hline $\begin{array}{l}\text { Ácido } \\
\text { sulfhídrico } \\
\left(\mathrm{H}_{2} \mathrm{~S}\right)\end{array}$ & $\begin{array}{lr}\text { Fumigación } & \text { de } \\
\text { invernaderos; } & \text { abonos; } \\
\text { pescadores; } & \text { descarga } \\
\text { de } & \text { pescado; } \\
\text { manipulación } & \text { de } \\
\text { alcantarillado. } & \end{array}$ & $\begin{array}{l}\text { Agricultura; } \\
\text { pesca; trabajo en } \\
\text { alcantarillas }\end{array}$ & $\begin{array}{l}\text { M: Bloqueo del metabolismo } \\
\text { oxidativo } \\
\text { A: Pérdida de conciencia } \\
\text { C: Encefalopatía }\end{array}$ \\
\hline $\begin{array}{l}\text { Cianuro } \\
(H C N)\end{array}$ & $\begin{array}{l}\text { Electrosoldadura; } \\
\text { galvanoplastia con } \\
\text { níquel; cobre y plata; } \\
\text { fumigación de barcos, } \\
\text { casas, alimentos y } \\
\text { suelos de invernaderos. }\end{array}$ & $\begin{array}{l}\text { Industria } \\
\text { metalúrgica; } \\
\text { industrias } \\
\text { químicas; viveros; } \\
\text { minería; fábricas } \\
\text { de gas. }\end{array}$ & $\begin{array}{l}\text { M: Bloqueo de enzimas } \\
\text { respiratorias } \\
\text { A: Disnea; descenso de la tensión } \\
\text { arterial; convulsiones; pérdida de } \\
\text { conciencia; muerte } \\
\text { C: Encefalopatía; ataxia; neuropatía. } \\
\text { Alteración profesional incierta }\end{array}$ \\
\hline $\begin{array}{l}\text { Óxido } \\
\text { Nitroso } \\
\left(\mathrm{N}_{2} \mathrm{O}\right)\end{array}$ & \begin{tabular}{lrr} 
Anestesia & \multicolumn{2}{r}{ general } \\
durante & \multicolumn{2}{c}{ cirugía; } \\
narcosis & ligera & en \\
asistencia & dental & y \\
parto. & &
\end{tabular} & $\begin{array}{l}\text { Hospitales } \\
\text { (anestesia); } \\
\text { dentistas; } \\
\text { comadronas }\end{array}$ & $\begin{array}{l}\text { M: Alteración aguda de la membrana } \\
\text { de la célula nerviosa; degeneración } \\
\text { de células nerviosas después de } \\
\text { exposición prolongada } \\
\text { A: Mareos; somnolencia; pérdida de } \\
\text { conciencia } \\
\text { C: Entumecimiento de los dedos de } \\
\text { manos y pies; disminución de } \\
\text { coordinación; encefalopatía. }\end{array}$ \\
\hline
\end{tabular}

M: mecanismos; A: efectos agudos; C efectos crónicos

Fuente: Enciclopedia de Salud y Seguridad en el trabajo, OIT, Tomo 1, Cap. 7, Pág.10,

Tabla 3. Disolventes orgánicos asociados a neurotoxicidad.

\begin{tabular}{|c|c|c|c|}
\hline $\begin{array}{l}\text { Producto } \\
\text { químico }\end{array}$ & $\begin{array}{l}\text { Ejemplos de fuentes } \\
\text { de exposición }\end{array}$ & $\begin{array}{c}\text { Selección de } \\
\text { industrias con } \\
\text { riesgo }\end{array}$ & Efectos \\
\hline $\begin{array}{l}\text { Hidrocarbonos } \\
\text { clorados: } \\
\text { Bicloroetileno; } \\
1,1,1- \\
\text { tricloroetano; } \\
\text { tetracloroetileno }\end{array}$ & $\begin{array}{l}\text { Desengrasado; } \\
\text { galvanización; pintura; } \\
\text { impresión; limpieza; } \\
\text { anestesia general y } \\
\text { ligera. }\end{array}$ & $\begin{array}{l}\text { Industria } \\
\text { metalúrgica; } \\
\text { industria gráfica; } \\
\text { industria } \\
\text { electrónica; } \\
\text { limpiezas en seco; } \\
\text { anestesistas. }\end{array}$ & $\begin{array}{l}\text { M: Desconocido } \\
\text { A: Síntomas prenarcóticos } \\
\text { C:Encefalopatía; polineuropatía; } \\
\text { afectación trigeminal (TRI); } \\
\text { pérdida de audición }\end{array}$ \\
\hline $\begin{array}{l}\text { Cloruro } \\
\text { metileno }\end{array}$ & $\begin{array}{l}\text { Extracción, incluida } \\
\text { de la extracción de } \\
\text { cafeína: decapante de } \\
\text { pinturas }\end{array}$ & $\begin{array}{l}\text { Industria } \\
\text { alimentaria; } \\
\text { pintora; industria } \\
\text { gráfica. }\end{array}$ & $\begin{array}{l}\text { M: Metabolismo } \longrightarrow \text { cO } \\
\text { A: Síntomas prenarcóticos: } \\
\text { coma } \\
\text { C: Encefalopatía }\end{array}$ \\
\hline $\begin{array}{l}\text { Cloruro } \\
\text { metilo }\end{array}$ & $\begin{array}{l}\text { Producción } \\
\text { reparación } \\
\text { frigoríficos. }\end{array}$ & $\begin{array}{l}\text { Producción de } \\
\text { frigoríficos; } \\
\text { industria del } \\
\text { caucho; industria } \\
\text { de los plásticos. } \\
\end{array}$ & $\begin{array}{l}\text { M: Desconocidos } \\
\text { A: Síntomas prenarcóticos: } \\
\text { pérdida de conciencia; muerte } \\
\text { C: Encefalopatía }\end{array}$ \\
\hline Tolueno & $\begin{array}{l}\text { Impresión; limpieza; } \\
\text { desengrasado; } \\
\text { galvanización; pintura; } \\
\text { pintura con pistola. }\end{array}$ & $\begin{array}{l}\text { Industria gráfica; } \\
\text { industria } \\
\text { electrónica. }\end{array}$ & $\begin{array}{l}\text { M: Desconocido } \\
\text { A: Síntomas prenarcóticos } \\
\text { C: Encefalopatía; disfunción } \\
\text { cerebelosa; polineuropatía; } \\
\text { pérdida de r audición; } \\
\text { polineuropatía; rastornos } \\
\text { visuales }\end{array}$ \\
\hline
\end{tabular}




\begin{tabular}{|c|c|c|c|}
\hline Xileno & $\begin{array}{lr}\text { Impresión } & \text { síntesis de } \\
\text { anhídrido } & \text { ftálico; } \\
\text { pinturas; } & \text { técnicas } \\
\text { histológicas } & \text { de } \\
\text { laboratorio. } & \end{array}$ & $\begin{array}{lr}\text { Industria } & \text { gráfica, } \\
\text { industria de } & \text { los } \\
\text { plásticos; } & \\
\text { laboratorios } & \text { de } \\
\text { histología. } & \end{array}$ & $\begin{array}{l}\text { M: Desconocido } \\
\text { A: Síntomas prenarcóticos } \\
\text { C: Encefalopatía; trastornos } \\
\text { visuales; pérdida polineuropatía } \\
\text { de audición }\end{array}$ \\
\hline Estireno & $\begin{array}{l}\text { Polimerización; } \\
\text { moldeado. }\end{array}$ & $\begin{array}{l}\text { Industria de los } \\
\text { plásticos; } \\
\text { producción de fibra } \\
\text { de vidrio. }\end{array}$ & $\begin{array}{l}\text { M: Desconocido } \\
\text { A: Síntomas prenarcóticos } \\
\text { C: } \quad \text { Encefalopatía; } \\
\begin{array}{l}\text { polineuropatía; pérdida de } \\
\text { audición }\end{array}\end{array}$ \\
\hline $\begin{array}{l}\text { Hexacartonos: } \\
\text { n-hexano; metil } \\
\text { butil cetona } \\
\text { (MBK); metil etil } \\
\text { cetona (MEK) }\end{array}$ & $\begin{array}{l}\text { Encolado; impresión, } \\
\text { revestimientos; } \\
\text { plásticos; pinturas; } \\
\text { extracción. }\end{array}$ & $\begin{array}{l}\text { Industria del cuero } \\
\text { y del calzado; } \\
\text { industria gráfica; } \\
\text { pintores; } \\
\text { laboratorios }\end{array}$ & $\begin{array}{l}\text { M: Deterioro del transporte } \\
\text { axonal } \\
\text { A: Síntomas prenarcóticos } \\
\text { C: Polineuropatía; encefalopatía }\end{array}$ \\
\hline $\begin{array}{l}\text { Disolventes } \\
\text { diversos: } \\
\text { Freón } 113\end{array}$ & $\begin{array}{lr}\text { Producción } & y \\
\text { reparación } & \text { de } \\
\text { frigoríficos; } & \text { limpieza } \\
\text { en } & \text { seco; } \\
\text { desengrasado. } & \end{array}$ & $\begin{array}{l}\text { Producción de } \\
\text { frigoríficos; } \\
\text { industria } \\
\text { metalúrgica; } \\
\text { industria } \\
\text { electrónica; } \\
\text { limpieza en seco }\end{array}$ & $\begin{array}{l}\text { M: Desconocido } \\
\text { A: Síntomas prenarcóticos leves } \\
\text { C: Encefalopatía }\end{array}$ \\
\hline $\begin{array}{l}\text { Dietiléter, } \\
\text { halotano }\end{array}$ & $\begin{array}{l}\text { Anestésicos } \\
\text { generales } \\
\text { (personal de } \\
\text { enfermería; médicos). }\end{array}$ & $\begin{array}{l}\text { Hospitales; } \\
\text { clínicas }\end{array}$ & $\begin{array}{l}\text { M: Desconocido } \\
\text { A: Síntomas prenarcóticos } \\
\text { C: Encefalopatía. }\end{array}$ \\
\hline $\begin{array}{l}\text { Disulfuro de } \\
\text { carbono }\end{array}$ & Véase monómeros & Véase monómeros & Véase monómeros \\
\hline $\begin{array}{l}\text { Mezclas: } \\
\text { aguarrás } \\
\text { diluyentes }\end{array}$ & $\begin{array}{l}\text { Pintura; } \\
\text { desengrasante; } \\
\text { limpieza; impresión; } \\
\text { impregnación; } \\
\text { tratamiento } \\
\text { superficial. }\end{array}$ & $\begin{array}{l}\text { Industria } \\
\text { metalúrgica; } \\
\text { industria gráfica; } \\
\text { industria de la } \\
\text { madera; pintores. }\end{array}$ & $\begin{array}{l}\text { M: Desconocido } \\
\text { A: Síntomas prenarcóticos } \\
\text { C: Encefalopatía. }\end{array}$ \\
\hline
\end{tabular}

M: mecanismos; A: efectos agudos; C: efectos crónicos

Fuente: Enciclopedia de Salud y Seguridad en el trabajo, OIT, Tomo 1, Cap. 7, Pág.12,

Tabla 4. Metales y sus compuestos inorgánicos asociados a neurotoxicidad.

\begin{tabular}{|c|c|c|c|}
\hline $\begin{array}{l}\text { Producto } \\
\text { químico }\end{array}$ & $\begin{array}{l}\text { Ejemplos de fuentes } \\
\text { de exposición }\end{array}$ & $\begin{array}{l}\text { Selección de } \\
\text { industrias } \\
\text { riesgo }\end{array}$ & Efectos \\
\hline Plomo & $\begin{array}{l}\text { Fundición; soldadura; } \\
\text { molienda; reparaciones; } \\
\text { vidriado; plastificado }\end{array}$ & $\begin{array}{l}\text { Metalurgia; } \\
\text { minería; fábricas } \\
\text { de acumuladores; } \\
\text { reparación de } \\
\text { coches; astilleros; } \\
\text { trabajadores del } \\
\text { vidrio; cerámica; } \\
\text { alfarería; plásticos }\end{array}$ & $\begin{array}{l}\text { M: Deterioro del metabolismo } \\
\text { oxidativo de células nerviosas y glía } \\
\text { A: Dolor abdominal; cefaleas; } \\
\text { encefalopatía; convulsiones. } \\
\text { C: Encefalopatía; polineuropatía; } \\
\text { incluida mano péndula }\end{array}$ \\
\hline $\begin{array}{l}\text { Mercurio } \\
\text { elemental }\end{array}$ & $\begin{array}{l}\text { Electrólisis, } \\
\text { instrumentos } \\
\text { electrónicos (giroscopio, } \\
\text { manómetro; }\end{array}$ & $\begin{array}{l}\text { Plantas de } \\
\text { clorcalcalinos; } \\
\text { minería; }\end{array}$ & $\begin{array}{l}\text { M: Deterioro en varios lugares de las } \\
\text { células nerviosas } \\
\text { A: Inflamación pulmonar, cefaleas; } \\
\text { trastornos del lenguaje }\end{array}$ \\
\hline
\end{tabular}




\begin{tabular}{|c|c|c|c|}
\hline & 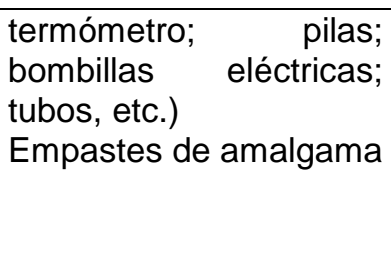 & $\begin{array}{l}\text { electrónica; } \\
\text { dentistas; } \\
\text { producción de } \\
\text { polímeros; } \\
\text { industria papelera } \\
\text { y de la celulosa. }\end{array}$ & $\begin{array}{l}\text { C: Inflamación de las encías; pérdida } \\
\text { de apetito; Encefalopatía; temblor; } \\
\text { irritabilidad. }\end{array}$ \\
\hline $\begin{array}{l}\text { Calomel } \\
\mathrm{Hg}_{2} \mathrm{Cl}_{2}\end{array}$ & & Laboratorios & $\begin{array}{l}\text { A: Toxidad aguda baja; efectos } \\
\text { tóxicos crónicos, véase más arriba }\end{array}$ \\
\hline $\begin{array}{l}\mathrm{Hg}_{2} \mathrm{Cl}_{2} \\
\text { sublimado }\end{array}$ & Desinfección. & $\begin{array}{l}\text { Hospitales; } \\
\text { clínicas; } \\
\text { laboratorios. }\end{array}$ & $\begin{array}{l}\text { M: Degeneración renal tubular y } \\
\text { glomenular aguda: muy tóxico incluso } \\
\text { en dosis orales pequeñas; letal hasta } \\
30 \mathrm{mg} \mathrm{kg} \text { de peso } \\
\text { C: Véase más arriba }\end{array}$ \\
\hline Manganeso & $\begin{array}{l}\text { Fundición (aleación de } \\
\text { acero); corte; soldadura } \\
\text { de acero; pilas }\end{array}$ & $\begin{array}{l}\text { Minas de } \\
\text { manganeso; } \\
\text { producción de } \\
\text { acero y aluminio; } \\
\text { industria } \\
\text { metalúrgica; } \\
\text { producción de } \\
\text { pilas; industrias } \\
\text { químicas; fábricas } \\
\text { de ladrillos. }\end{array}$ & $\begin{array}{l}\text { M: Desconocido, ‘posibles cambios } \\
\text { de dopamina y cabecolaminas en los } \\
\text { ganglios basales del cerebro } \\
\text { A: Distrofia } \\
\text { C: Encefalopatía; incluido } \\
\text { parkinsonismo; psicosis; pérdida de } \\
\text { apetito, irritabilidad, cefaleas, } \\
\text { debilidad }\end{array}$ \\
\hline Aluminio & $\begin{array}{l}\text { Metalurgia; molineda } \\
\text { pulimentado }\end{array}$ & Industria del metal. & $\begin{array}{l}\text { M: Desconocido } \\
\text { C: Posible encefalopatía }\end{array}$ \\
\hline
\end{tabular}

M: mecanismos; A: efectos agudos; C: efectos crónicos

Fuente: Enciclopedia de Salud y Seguridad en el trabajo, OIT, Tomo 1, Cap. 7, Pág.11,

\section{Vías de Ingreso}

Las vías de ingreso implicadas en la acción tóxica de estas sustancias son: por inhalación de los gases o vapores presentes en el medio ambiente laboral, por contacto con la piel y en forma líquida por ingestión accidental o voluntaria siendo absorbidos a través del tracto digestivo. (Nolla. Et.al., 2001)

\section{Metabolismo de los Solventes Orgánicos}

Se analiza el metabolismo de los solventes orgánicos porque como se comentó anteriormente este trabajo tiene especial interés en este tipo de sustancias por sus características toxicológicas y su uso en diferentes sectores productivos, dado que están presentes en mezcla formando parte de muchos productos como adhesivos (tolueno solvente en el cemento de contacto), removedores y disolventes de pintura (tiñer), el tolueno se adiciona como antidetonante en los combustibles, se generan como producto de la combustión de la gasolina en los automotores, en la quema de bosques en los incendios forestales, en productos de limpieza, desengrasantes industriales y domésticos, etc. (Espinosa, 2008) 
Ingreso: La vías de ingreso de lo solventes son: Vía respiratoria (inhalación) y vía cutánea (absorción a través de la piel). La captación por inhalación es la principal vía y depende de "la concentración del disolvente en el aire inhalado, del coeficiente sangre/aire del disolvente (que es determinado por la variabilidad individual de la permeabilidad de la membrana alveolo-capilar y la solubilidad en sangre), la tasa de ventilación alveolar, la perfusión pulmonar y la duración de la exposición" (OMS, 1985; Astrand, 1975).

La actividad física tiene una relación directa con la concentración de tóxico inhalado, ya que al aumentar la actividad física aumenta la ventilación pulmonar y el ritmo cardíaco, aumentando la absorción pulmonar del disolvente. (Castellar, M., 2007).

Distribución. Después de la absorción, los disolventes orgánicos se distribuyen por vía sanguínea a todo el organismo una parte se metaboliza y otra se acumula en los tejidos ricos en lípidos, como los del sistema nervioso central y periférico, la médula ósea, bazo y glándulas suprarrenales.

Metabolismo. Es un proceso de transformación que se produce principalmente en el hígado, de acuerdo con Castellar M (2007) en su investigación en trabajadores del sector petrolero, los solventes orgánicos durante el metabolismo son transformados en compuestos solubles en agua que se eliminan a través de la orina, algunos de estos metabolitos pueden ser más tóxicos que las sustancias originales. También indica que una ingesta aguda de etanol aumenta la concentración de tolueno y xileno en la sangre, produciéndose un efecto sinérgico, esto se debe a que el etanol inhibe el metabolismo de estas sustancias (Cárdenas, O., Varona, M., Patiño, R., Groot, H., Sicard, D., Tórrez, M., \& Pardo, D., 2007).

Los metabolitos de BTX son utilizados como índices biológicos y permiten determinar el nivel de exposición de los trabajadores a estas sustancias. (Tabla 5)

Tabla 5.Índices Biológicos de exposición (BEI) para Benceno, Tolueno y Xileno (BTX).

\begin{tabular}{|l|l|l|}
\hline $\begin{array}{l}\text { Producto } \\
\text { químico }\end{array}$ & \multicolumn{1}{|c|}{ Metabolito } & $\begin{array}{l}\text { Índice biológico } \\
\text { de (BEI) }\end{array}$ \\
\hline Benceno & $\begin{array}{l}\text { Acido S-fenil mercaptúrico } \\
\text { en orina } \mu \mathrm{g} / \mathrm{g} \text { creatinina }\end{array}$ & $25 \mu \mathrm{g} / \mathrm{g}$ creatinina \\
\hline Tolueno & $\begin{array}{l}\text { Acido hipúrico en orina } \mathrm{g} / \mathrm{g} \\
\text { creatinina }\end{array}$ & $2,5 \mathrm{~g} / \mathrm{g}$ creatinina \\
\hline Xileno & $\begin{array}{l}\text { Acido metilhipúrico en orina } \\
\mathrm{g} / \mathrm{g} \text { creatinina }\end{array}$ & $1,5 \mathrm{~g} / \mathrm{g}$ creatinina \\
\hline
\end{tabular}

Fuente: American Conference of Governmental Industrial Hygienists (ACGIH). (2011). Introduction to the biologically derived airborne contaminants. TLVs and BEls. pp. 100-103.

El conjunto de síntomas que evidencian el daño del Sistema Nervioso Central se conoce como Síndrome Neurotóxico. Estos pueden ser: 
- Agudos, se producen en situaciones accidentales, en este caso desaparecen con rapidez si la exposición ha sido a breves periodos de tiempo.

- Crónicos, derivan en enfermedades profesionales debido a que se producen alteraciones neurológicas y morfológicas irreversibles, esto se da cuando la exposición ha bajas dosis ha sido frecuente a lo largo de su vida laboral, tomando en cuenta que el daño provocado por estas sustancias cada vez que ingresan al organismo es acumulativo.

\section{Consideraciones concluyentes}

El avance tecnológico y científico del mundo y su globalización, son los principales factores para la utilización de sustancias que producen efectos nocivos en los seres vivos y en el medio ambiente, siendo cada vez más habituales en las labores cotidianas, lo que lleva al trabajador a familiarizarse con ellas y muchas veces desestima el riesgo porque desconoce la magnitud de sus efectos.

En el Ecuador cientos de trabajadores están expuestos permanentemente a la contaminación neurotóxica en las diferentes industria sean estas públicas o privadas, sin embargo no existen políticas que prioricen la seguridad industrial, siendo de vital importancia realizar esta investigación para implementar planes y programas de prevención.

En este tema, la Salud, Seguridad e Higiene del trabajo tiene un amplio campo de estudio.

\section{Bibliografía}

Agencia para Sustancias Tóxicas y el Registro de Enfermedades. (ATSDR). 2001. Reseña Toxicológica del Tolueno (en inglés). Atlanta, GA: Departamento de Salud y Servicios Humanos de los EE.UU., Servicio de Salud Pública. Recuperado 28 de Noviembre 2013. http://www.atsdr.cdc.gov/es/toxfaqs/es_tfacts56.pdf

American Conference of Governmental Industrial Hygienists (ACGIH). (2011). Introduction to the biologically derived airborne contaminants. TLVs and BEls. (7ma ed.). Cincinnati, United States. Eds ACGIH. 100-103.

Almirall. P, Franco. G, Martínez. S, Noriega. M, Villegas. J, Mendez. I, "Evaluación psicológica en trabajadores expuestos a tolueno en una empresa mexicana de autopartes". Salud de los Trabajadores / Volumen 7 № 1 / Enero 1999. Recuperado 10 de Abril de 2012. http://www.bvsde.paho.org/bvsast/e/fulltext/tolueno/tolueno.pdf

Arlien-Soborg, P., Enciclopedia y Seguridad en el Trabajo. Sistema Nervioso. Agentes Químicos $\begin{array}{lllll}\text { Neurotóxicos. } & \text { Recuperado } & 6 & \text { de } & \text { septiembre }\end{array}$ http://www.insht.es/InshtWeb/Contenidos/Documentacion/TextosOnline/EnciclopediaOIT/tom 01/7.pdf 
Borgel, L., (2011), La gestión ambiental y seguridad química, paradigmas hacia la sustentabilidad empresarial. Seguridad y Salud en el Trabajo, Edición N³, Revista electrónica del IESS, $\begin{array}{lllll}\text { Quito. } & \text { Recuperado el de } 29 \text { de }\end{array}$ http://www.iess.gob.ec/multimedias/revista_digital/ver3/

Cárdenas, O., Varona, M., Patiño, R. I., Groot, H., Sicard, D., M Tórres, M., Pardo, D. (2007). Bogotá paint-industry workers exposure to organic solvents and genotoxic effects. Revista de Salud Pública, 9(2), 275-288. Recuperado el 27 de Noviembre de 2013. http://www.scielo.org.co/pdf/rsap/v9n2/v9n2a11.pdf

Carvajal, Y., Apuntes Técnicos sobre Enfermedades Profesionales- Subdepto. Salud en el Trabajo Sesma. Chile. Abril 2001. Recuperado el 13 de septiembre 2013. http://www.asrm.cl/Archivos/Servicios/estudio_manual_enf_prof.pdf

Castellar, M. "Efectos crónicos neurocomportamentales en trabajadores del sector petrolero expestos a solventes orgánicos aromáticos (benceno, tolueno y xileno- btx)", Tesis Especialista, Pontificia Universidad Javeriana, Bogota, 2007. Recuperado el 25 de Abril de 2012. http://www.javeriana.edu.co/biblos/tesis/enfermeria/tesis41.pdf

Centers for Disease Control and Prevention, Guía de bolsillo de NIOSH sobre riesgos químicos, Publicaciones National Institute for Occupational Safety and Health (NIOSH), Recuperado el 25 de Abril de 2012. http://www.cdc.gov/spanish/niosh/npg-sp/npg-sp.html

Cortés, J.M., (2007), Seguridad e Higiene del Trabajo, 9 Edición actualizada, Editorial Tébar S.L., Madrid. Pp 380-388, 635

Espinosa, M., (2008), Informe final del análisis y revisión de información que sustente la elaboración de una NOM sobre Benceno, Tolueno y Xileno, Secretaría de Medioambiente y Recursos Naturales, México. Recuperado el 29 de Noviembre del 2013. http://es.scribd.com/doc/13906292/Informe-Final-Dra-Espinosa

Gherardi, M., Gordiani A., Proietto, A., (2007), Medidas de la exposición a químicos en Restauración de obras de arte, Journal of Chemical Healt and Safety, Volumen 14. $\begin{array}{llllll}\text { Recuperado } & \text { el } & 28 & \text { de }\end{array}$ http://www.sciencedirect.com/science/article/pii/S1871553207000382.

Harari, R. t cols, Corp. IFA, (2007). DMQ-Ambiente y salud, Dirección Metropolitana de Medio Ambiente Quito - Corporación IFA, publicación en línea. Recuperado el 10 de Julio de 2012. http://www.ifa.org.ec/DMQ_AMBIENTEYSALUD2.pdf 
Harari, R. t cols, Corp. IFA. (2007). Seguridad, Salud y Ambiente en la floricultura, Corporación IFA, publicación en línea. Recuperado el 10 de Julio de 2012. http://ifa-orgec.ambienteysalud.org/floricultura.pdf

Instituto Ecuatoriano de Seguridad Social IESS, (2011), Resolución D.C. 390

Instituto Nacional para la Seguridad y Salud Ocupacional (NIOSH), Esfuerzo de campo de NIOSH para evaluar los riesgos de la exposición a sustancias químicas en los trabajadores de la industria de extracción de petróleo y gas, Publicación de NIOSH No. 2010-130. Recuperado el 23 de Julio de 2012. http://www.cdc.gov/spanish/niosh/docs/2010-130_sp/.

Maizlish, N. Feo, O. "Alteraciones neuropsicológicas en trabajadores expuestos a neurotóxicos". Salud de los Trabajadores / Volumen 2 № 1 / Enero 1994. Recuperado el 10 de Abril de 2012. http://www.segurancaetrabalho.com.br/download/alteraciones-oscar-feo.pdf

Mayor. J, "Efectos de los solventes orgánicos sobre el sistema nervioso. los métodos neurológicos". Salud de los Trabajadores I Volumen 3 NN$^{\circ} 2$ / Julio 1995. Recuperado el 10 de Abril de 2012. www.higieneocupacional.com.br/download/efectos-jorge-rios.pdf

Enciclopedia OIT, Salud y Seguridad en el Trabajo. Sistema Nervioso (Mergler, D., Directora del capítulo). Tomo 1, Cap. 7, pp 1-29. Recuperado el 6 de septiembre 2013. http://www.insht.es/InshtWeb/Contenidos/Documentacion/TextosOnline/EnciclopediaOIT/tom 01/7.pdf

Nolla, M. H. J., Martín, J. C., Yetamos, F., Martínez-Coronel, J. F., Bellot, J. L., Ferrer, A., ... \& López-Álvaro, J. (2001). Intoxicaciones agudas II. Tratado de cuidados críticos y emergencias, 1501. Recuperado el 5 de Septiembre de 2013. http://books.google.es/books?hl=es\&lr=\&id=-

bZQZQhitGYC\&oi=fnd\&pg=PA1501\&dq=SIDROME+NEUROT\%C3\%93XICO+POR+EXPOS ICI\%C3\%93N+A+SOLVENTES+ORG\%C3\%81NICOS\&ots=Dp1-

rmu_xB\&sig=6nVdIUJ6fNKQvFuj1TKAaWB8z5A\#v=onepage\&q\&f=false

Oficina Internacional de Trabajo OIT, (2010), Lista de enfermedades profesionales (revisada 2010), Serie Seguridad y Salud en el Trabajo 74, Recuperado el 10 de Julio de 2012. http://www.ilo.org/wcmsp5/groups/public/---ed_protect/---protrav/--safework/documents/publication/wcms_150327.pdf

Olmos, M., (2011), S ustancias Químicas Peligrosas, Manual de Seguridad en el Trabajo, Fundación MAPFRE, $2^{\circ}$ edición, España, pp 977-1074 
Rimblas, M., Consejería de Sanidad. Los Compuestos Químicos en los Alimentos desde la perspectiva de la Seguridad Alimentaria. Región de Murcia. Recuperado el 10 de septiembre 2013. http://www.murciasalud.es/recursos/ficheros/82501-alimentos.pdf

Rodríguez, G. A. (2011). Conflictos ambientales amenazan la salud de la población y la biodiversidad del planeta. Revista de Derecho, 28(28). Recuperado el 25 de Septiembre de 2013 http://rcientificas.uninorte.edu.co/index.php/derecho/article/viewArticle/2676

Rodríguez, E., (1999),Síndromes neurotóxicos causados por exposición a plaguicidas, Ponencia Reunión Sociedad Catalana de Medicina del Trabajo, Barcelona. Recuperado 5 de septiembre 2013. http://www.asquifyde.es/uploads/documentos/Sindromes-neurotoxicospor-exposici\%C3\%B3n-a-plaguicidas-\%20E.R.Farr\%C3\%A9-1999.pdf

Sacristan, R. (20039 "Toxicología de los materiales pictóricos", Tesis doctoral, Universidad Complutense de Madrid. España. Recuperado el 25 de Septiembre de 2013. http://www.ucm.es/BUCM/tesis/19972000/H/1/H1014501.pdf

Simonsen L, Johnsen H, Lund SP, Matikainen E, Midtgård U, Wennberg A., (1994), Methodological approach to the evaluation of neurotoxicity data and the classification of neurotoxic chemicals, Recuperado el 5 de Septiembre de 2013. http://www.ncbi.nlm.nih.gov/pubmed/8016593

Vela, M.M., Laborda, R. Y García, A.M., en Neurotóxicos en el ambiente laboral: criterios de clasificación y listado provisional. Arch prev Riesgos Labor 2003; 6 (1): 17-25. Recuperado el 20 de septiembre 2013. http://www.istas.net/risctox/index.asp?idpagina=611 